\title{
Prediction of renal outcome in Henoch-Schönlein nephritis based on biopsy findings
}

\author{
Mikael Koskela ${ }^{1,2} \cdot$ Elisa Ylinen ${ }^{2} \cdot$ Helena Autio-Harmainen $^{3} \cdot$ Heikki Tokola $^{3} \cdot$ Päivi Heikkilä $^{4} \cdot$ Jouko Lohi $^{4}$. \\ Hannu Jalanko ${ }^{2} \cdot$ Matti Nuutinen ${ }^{5,6} \cdot$ Timo Jahnukainen $^{2}$
}

Received: 13 June 2019 / Revised: 5 October 2019 / Accepted: 1 November 2019 / Published online: 3 December 2019

(C) The Author(s) 2019

\begin{abstract}
Background In Henoch-Schönlein nephritis (HSN), a risk factor for unfavorable outcome is prolonged proteinuria, but the value of renal biopsies in prognosis assessment is debatable.

Methods We evaluated serial renal biopsies from 26 HSN patients. Follow-up biopsy occurred at median 2.1 years after diagnostic biopsy. Patients formed two groups at the follow-up biopsy: patients without proteinuria (group I; $n=11$ ) and with proteinuria (group II; $n=15$ ). Biopsies underwent evaluation according to three classifications: International Study of Kidney Disease in Children (ISKDC), Oxford (MEST-C), and semiquantitative classification (SQC) including an activity and chronicity score. Analysis also included expression of pro-fibrotic (alpha-smooth muscle actin and vimentin) and inflammatory (P-selectin glycoprotein ligand-1) molecules in the diagnostic biopsy specimens. Definition of unfavorable outcome was active renal disease or reduced renal function at last follow-up.

Results Between the biopsies, SQC chronicity score increased in $22(85 \%)$ patients, whereas activity score and ISKDC grade decreased in $21(81 \%)$ and 17 (65\%), respectively. Of the MEST-C parameters, endocapillary proliferation (from 83 to $13 \% ; p<$ 0.001 ) and crescents (from 63 to $25 \% ; p=0.022$ ) showed significant reduction, and segmental glomerulosclerosis (from 38 to $79 \% ; p=0.006$ ) significant increment. These changes occurred similarly in groups I and II. Expression of the pro-fibrotic and inflammatory molecules showed no clinically significant differences between groups I and II. None in group I and five (33\%) patients in group II had unfavorable outcome $(p=0.053)$.

Conclusions Our results suggest that follow-up biopsies provide limited additional information to clinical symptoms in HSN outcome prediction.
\end{abstract}

Keywords $\alpha$-SMA $\cdot$ Vimentin $\cdot$ PSGL-1 $\cdot$ Semiquantitative $\cdot$ Oxford classification $\cdot$ Immunohistochemistry

\section{Introduction}

The most common vasculitis in children is Henoch-Schönlein purpura (HSP) [1]. Its typical symptoms manifest in the skin,

Electronic supplementary material The online version of this article (https://doi.org/10.1007/s00467-019-04415-3) contains supplementary material, which is available to authorized users.

Mikael Koskela

mikael.koskela@helsinki.fi

1 Children's Hospital, Pediatric Research Center, University of Helsinki, Helsinki University Hospital, Helsinki, Finland

2 Department of Pediatric Nephrology and Transplantation, New Children's Hospital, University of Helsinki and Helsinki University Hospital, PO Box 347, Stenbäckinkatu 9, 00029 Helsinki, Finland gastro-intestinal tract, joints, and kidneys, and the severity of the renal symptoms determines the long-term outcome. Of HSP patients, on average, 34\% develop renal symptoms [2]. The symptoms are typically self-limiting proteinuria or
3 Medical Research Center Oulu and Department of Pathology, Oulu University Hospital, Oulu, Finland

4 Department of Pathology, Helsinki University Hospital, Helsinki, Finland

5 Department of Children and Adolescents, Oulu University Hospital, Oulu, Finland

6 PEDEGO Research Unit, Research Unit for Pediatrics, Dermatology, Clinical Genetics, Obstetrics and Gynecology, Medical Research Center Oulu (MRC Oulu), Oulu, Finland 
hematuria, or both, but some patients develop severe proteinuria, and these patients are at greater risk for chronic kidney disease [3, 4].

For Henoch-Schönlein nephritis (HSN), the most common histologic classification is from the International Study of Kidney Disease in Children (ISKDC) [5]. Although it also considers mesangial proliferation, ISKDC classification is largely based on the percentage of glomeruli with crescents, and treatment modality selection is based on ISKDC grade. The prognostic value of the ISKDC classification is, however, questionable, giving rise to a need for a new classification [6, 7]. The Oxford classification for IgA nephropathy (IgAN), a disease with similar renal histology to HSN, appeared in 2009 [8]. The updated version of the Oxford classification (MESTC) now considers five histologic parameters: mesangial hypercellullarity (M), endocapillary proliferation (E), segmental glomerulosclerosis (S), tubular atrophy and/or interstitial fibrosis (T), and crescents (C) [9]. However, in pediatric HSN, the feasibility of MEST-C requires still further research [7]. Furthermore, we recently introduced a semiquantitative classification (SQC) for histologic evaluation of HSN [10].

In this study, we evaluated ISKDC, SQC, and MEST-C scores in sequential renal biopsy specimens. Additionally, from diagnostic kidney biopsies, we analyzed immunohistochemically the expression of pro-fibrotic and inflammatory cell markers. Alpha-smooth muscle actin ( $\alpha$-SMA) and vimentin are pro-fibrotic markers, whereas P-selectin glycoprotein ligand-1 (PSGL-1) is a marker for inflammatory cells; early detection of the processes featuring these markers could prove important in enabling early treatment before the development of fibrosis. The aim of our study was to evaluate the prognostic value of (1) the ISKDC, SQC, and MEST-C classifications and (2) the expression of pro-fibrotic and inflammatory markers. Our hypothesis was that expression of these markers in the diagnostic kidney biopsies correlates with the findings in the follow-up renal biopsy, and that their expression is higher in HSN patients than in control samples from normal kidneys.

\section{Materials and methods}

\section{Study design}

The study comprises 26 childhood-onset ( $<17$ years) patients with HSN with an available follow-up renal biopsy specimen from Oulu University Hospital (1985-2005), Helsinki University Hospital (2000-2010) and a nationwide study [11]. A detailed description of the study population exists in our previous study, which analyzed the role of diagnostic renal biopsies for predicting outcomes in 53 HSN patients [10]. This study was approved by the ethics committee of Helsinki University Hospital (164/13/03/03/2016).

\section{Clinical data}

Definition of proteinuria was urine protein to creatinine ratio (UP/C) $>20 \mathrm{~g} / \mathrm{mol}$, daily urine protein excretion (dU-Prot) > $200 \mathrm{mg}$, or a positive urine dipstick test $(1+$ to $3+)$. UP/C values at the time of the renal biopsies were converted into dU-Prot [12], and values over $40 \mathrm{mg} / \mathrm{h} / \mathrm{m}^{2}$ denoted nephroticrange proteinuria. Definition of hematuria was $>20$ red blood cells/10E6/L or a positive urine dipstick test $(1+$ to $3+)$. Glomerular filtration rate was estimated (eGFR) with the bedside Schwartz equation [13]. Medical records provided data from the time of the diagnostic and follow-up biopsies and the last follow-up. Time from the onset of nephritis (proteinuria and/or hematuria) to renal biopsy and initiation of treatment was calculated. Patients $(n=2)$ who had not received immunosuppressive therapy were not included in the treatment delay analyses. Follow-up time was the period from HSPdiagnosis to the latest follow-up visit or to the start of renal replacement therapy. Indication for the diagnostic renal biopsy was either nephrotic-state proteinuria or persistence of proteinuria and/or hematuria up to 6-8 weeks. The 26 patients formed two groups at follow-up renal biopsy: patients without proteinuria (group I; $n=11$ ) and with proteinuria (group II; $n=$ 15). Eleven patients had no proteinuria at follow-up biopsy: nine of them underwent follow-up biopsy as part of a previous trial in accordance with the study protocol [11], one due to hematuria, and one for control purposes.

\section{Outcome}

Outcome assessment at the last follow-up was as follows: outcome A (healthy) — no signs of renal disease; outcome B (minor urinary abnormalities) - UP/C $=20-100 \mathrm{~g} / \mathrm{mol}$ and $/$ or microscopic hematuria and/or ongoing ACE-I treatment; outcome $\mathrm{C}$ (active renal disease) - UP/C > $100 \mathrm{~g} / \mathrm{mol}$ and/or ongoing immunosuppressive treatment; outcome D (reduced renal function) - $\mathrm{eGFR}<60 \mathrm{~mL} / \mathrm{min} / 1.73 \mathrm{~m}^{2}$. Outcomes A + $\mathrm{B}$ were categorized as favorable outcome and outcomes $\mathrm{C}+\mathrm{D}$ as unfavorable outcome.

\section{Renal biopsy classifications}

Renal pathologists blinded to the patients' medical history reevaluated the biopsies with the ISKDC classification, SQC, and MEST-C. A detailed explanation of SQC parameters exists in our previous study [10]; the classification is also visible in online Table S1. Briefly, SQC comprises 14 renal histologic parameters and has a maximum score of 26 points; it divides into activity (maximum 9 points) and chronicity indices (maximum 16 points). In addition, a tubulointerstitial (including all active and chronic tubular and interstitial parameters) index can be calculated (maximum 5 points). The MEST-C scoring system of the Oxford classification includes five parameters 
and is defined as follows: M (mesangial hypercellularity defined as more than four mesangial cells in any mesangial area) as M0 ( $<50 \%$ of glomeruli with mesangial hypercellularity) or M1 (> 50\%); E (endocapillary proliferation) as E0 (absent) or E1 (present); S (segmental glomerulosclerosis) as S0 (absent) or S1 (present); T (tubular atrophy and/or interstitial fibrosis) as T0 (0-25\% of cortical area affected), T1 (26$50 \%)$, or $\mathrm{T} 2(>50 \%)$ and $\mathrm{C}$ (crescents) as $\mathrm{C} 0$ (absent), $\mathrm{C} 1$ (at least 1 crescent, but crescents in a maximum of $25 \%$ of glomeruli) or $\mathrm{C} 2(>25 \%)$. In addition, total MEST-C score was calculated (sum of all five MEST-C parameters).

\section{Immunohistochemistry and microscopy}

Diagnostic renal biopsy specimens, formalin-fixed and paraffin-embedded, were cut into $4-5-\mu \mathrm{m}$-thick slices. They underwent a conventional immunohistochemical staining process. Primary antibodies were used against $\alpha$-SMA (clone 1A4, diluted 1:400, Dako Denmark A/S, Glostrup, Denmark), vimentin (clone 3B4, 1:200, Dako), and PSGL-1 (sc-13535, 1:500, Santa Cruz Biotechnology, Inc., Dallas, TX, USA). Eighteen (69\%) biopsies were successfully stained with $\alpha$-SMA, 19 (73\%) with vimentin, and $17(65 \%)$ with PSGL-1. Negative controls containing no primary antibodies were incubated in phosphate-buffered saline. Normal kidneys, originally removed with an intent to use as kidney transplants, served as control specimens. Supplementary material contains images (Figures S1-S3) of typical expression of the analyzed molecules in HSN patients and in control specimens.

The microscopy tool used was Zeiss AX10. Analyses of the HSN biopsy specimens involved all glomeruli (with $\times 20$ magnification) and as many microscopic fields as possible from the cortical tubulointerstitium $(\times 40)$. Analysis of each control specimen included 30 randomly selected glomeruli $(\times 20)$ and 30 randomly selected, non-overlapping tubulointerstitial microscopic fields $(\times 40)$. Zeiss Zen software (Carl Zeiss AG, Oberkochen, Germany) performed calculation of the tubulointerstial area of positive $\alpha$-SMA and vimentin staining. The number of PSGL-1 staining patches was counted from the tubulointerstitium and glomeruli. In evaluation of the staining results, scores of all microscopic fields and glomeruli were totaled separately for control specimens, group I, and group II. To assess correlation with staining results and biopsy findings in HSN patients, mean of the microscopic fields' affected areas ( $\alpha$-SMA, vimentin) and the mean number of staining patches in the tubulointerstitium and glomeruli (PSGL-1) represented the scores for each patient. Patients' clinical data was not available when conducting the immunohistochemical analyses.

\section{Statistical analyses}

The statistical analysis software used was IBM SPSS version 22 (IBM Corp, Armonk, NY). Continuous variables are reported as means and standard deviations (SD) (normal distribution) or medians and interquartile ranges (IQR) (non-normal distribution), and analyzed with Student's $t$ test (normal distribution) or Mann-Whitney $U$ test (non-normal distribution). Categorical variables are reported as numbers and percentages, and analyzed with Fisher's exact test. Change in histologic variables from the diagnostic biopsy to the followup biopsy was analyzed with McNemar test (MEST-C parameters) or Wilcoxon-signed rank test (SQC parameters). Spearman rank correlations were calculated between staining results, biopsy findings, and biopsy delays. Results from linear regression are reported as regression coefficients ( $B$ value) with $95 \%$ confidence intervals. Predictors with $p<0.2$ in univariable analysis were selected to the multivariable analysis. The level of statistical significance was $p<0.05$.

\section{Results}

\section{Patient characteristics and outcome}

Table 1 provides baseline patient characteristics. Patients in group II were older than those in group 1; no other statistically significant differences existed between the groups at the time of the diagnostic renal biopsy. Median time from onset of nephritis to the diagnostic renal biopsy was 59 (IQR 36-71) days in patients without proteinuria (group I) and 33 (IQR 19$60)$ days in proteinuric patients (group II) $(p=0.17)$. Median time from renal biopsy to immunosuppressive treatment was 7 (IQR 4-8) days and 7 (IQR 2-16) days in groups I and II, respectively $(p=0.94)$.

Table 2 presents patient characteristics at the follow-up biopsy and at last follow-up. Follow-up biopsy was performed a median of 2.2 (IQR 2.0-2.3) years after the diagnostic biopsy in group I and $2.1(1.7-2.2)$ years in group II $(p=0.31)$. At the follow-up biopsy, plasma creatinine levels were significantly higher in group II than in group I, but there was no statistically significant difference in eGFR. Clinical and biopsy characteristics according to the outcome (favorable/unfavorable) are presented in the Supplementary material (Table S2). At the followup biopsy, patients with unfavorable outcome had significantly higher proteinuria than those with favorable outcome (median dU-Prot 4.7 vs. $0.2 \mathrm{~g} /$ day; $p<0.001$ ).

At the last follow-up, patients without proteinuria (group I) had significantly lower creatinine and higher eGFR than proteinuric patients (group II). Fifteen (58\%) patients belonged to outcome group A, 6 (23\%) to outcome group B, 1 (4\%) to outcome group C, and 4 (15\%) to outcome group D. Five patients had unfavorable outcome: one had ongoing cyclosporine A treatment due to persistent proteinuria, one had reduced renal function (eGFR $37 \mathrm{~mL} / \mathrm{min} / 1.73 \mathrm{~m}^{2}$ ), two developed endstage kidney disease, and one had succumbed due to HSN [14]. 
Table 1 Baseline characteristics at the diagnostic renal biopsy, ISKDC findings of the diagnostic biopsy, and used therapies

\begin{tabular}{|c|c|c|c|c|}
\hline & All $(n=26)$ & Group I $(n=11)$ & Group II $(n=15)$ & $p$ value \\
\hline Male, $n(\%)$ & $17(65 \%)$ & $5(45 \%)$ & $12(80 \%)$ & 0.10 \\
\hline Age (years) & $9.8 \pm 3.4$ & $7.7 \pm 2.6$ & $11.2 \pm 3.1$ & 0.006 \\
\hline Plasma creatinine $(\mu \mathrm{mol} / \mathrm{L})$ & $52(45-58)$ & $51(44-55)$ & $53(46-62)$ & 0.30 \\
\hline eGFR $\left(\mathrm{mL} / \mathrm{min} / 1.73 \mathrm{~m}^{2}\right)$ & $99(87-109)$ & $90(87-108)$ & $100(89-108)$ & 0.80 \\
\hline Plasma albumin $(\mathrm{g} / \mathrm{L})$ & $29.3 \pm 8.8$ & $30.4 \pm 8.2$ & $28.7 \pm 9.4$ & 0.66 \\
\hline dU-Prot ${ }^{\mathrm{a}}(\mathrm{g} /$ day $)$ & $4.8(1.6-7.7)$ & $3.8(1.0-5.8)$ & $4.8(1.8-10.2)$ & 0.50 \\
\hline dU-Prot ${ }^{\mathrm{a}}\left(\mathrm{mg} / \mathrm{h} / \mathrm{m}^{2}\right)$ & $140(69-328)$ & $136(52-233)$ & $150(77-332)$ & 0.72 \\
\hline Nephrotic-range proteinuria $\left(>40 \mathrm{mg} / \mathrm{h} / \mathrm{m}^{2}\right), n(\%)$ & $20(77 \%)$ & $8(73 \%)$ & $12(80 \%)$ & $>0.99$ \\
\hline Hematuria, $n(\%)$ & $26(100 \%)$ & $11(100 \%)$ & $15(100 \%)$ & $>0.99$ \\
\hline \multicolumn{5}{|l|}{ ISKDC } \\
\hline I & 0 & 0 & 0 & \\
\hline II & 6 & 4 & 2 & \\
\hline IIIa & 5 & 1 & 4 & \\
\hline IIIb & 12 & 6 & 6 & \\
\hline IV & 2 & 0 & 2 & \\
\hline $\mathrm{V}$ & 1 & 0 & 1 & \\
\hline SQC activity score & $4(2-7)$ & $4(1-4)$ & $6(4-7.5)$ & 0.018 \\
\hline SQC chronicity score & $3(2-4)$ & $3(1.5-3.5)$ & $3(2.5-4)$ & 0.37 \\
\hline SQC tubulointerstitial score & $0(0-1)$ & $0(0-0.5)$ & $0(0-1)$ & 0.31 \\
\hline $\mathrm{IT}, n(\%)$ & $24(92 \%)$ & $9^{\mathrm{b}}(82 \%)$ & $15^{\mathrm{c}}(100 \%)$ & 0.17 \\
\hline ACE-I, $n(\%)$ & $21(81 \%)$ & $8(73 \%)$ & $13(87 \%)$ & 0.62 \\
\hline
\end{tabular}

Group I represents patients without proteinuria and group II patients with proteinuria at the follow-up biopsy. eGFR, estimated glomerular filtration rate; $d U$-Prot, daily urine protein excretion; IT, immunosuppressive therapy; ACE-I, angiotensin-converting enzyme inhibitor

${ }^{a}$ For one patient in group I proteinuria at the diagnostic renal biopsy was measured only as a negative dipstick test and was not included in the analyses

${ }^{\mathrm{b}}$ Initial immunosuppressive therapies in group I were methylprednisolone pulses $(n=6)$ and cyclosporine A $(n=3)$. Two patients had not received immunosuppressive therapy

${ }^{\mathrm{c}}$ Initial immunosuppressive therapies in group II were methylprednisolone pulses $(n=8)$, cyclosporine $\mathrm{A}(n=4)$, azathioprine $(n=1)$, cyclophosphamide $(n=1)$, and oral prednisone $(n=1)$

None in group I developed unfavorable outcome compared to five patients $(33 \%)$ in group II $(p=0.053)$.

\section{SQC and ISKDC}

In the diagnostic and follow-up biopsies of all 26 patients, median activity scores were 4 (IQR 2-7) and 2 (IQR 1-2) ( $p$ $<0.001$ ), median chronicity scores 3 (IQR 2-4) and 7 (IQR 59) $(p<0.001)$, and median tubulointerstitial scores 0 (IQR 0 $1)$ and 3 (IQR 2-3) $(p<0.001)$, respectively. Activity scores in the diagnostic and follow-up biopsy and chronicity score in the follow-up biopsy were significantly higher in group II than in group I (Tables 1 and 2). Figure 1 illustrates individually in each patient the changes in the SQC activity, chronicity, and tubulointerstitial scores, and in the ISKDC grades between diagnostic and follow-up biopsy. Between the biopsies, SQC activity score decreased in $21(81 \%)$ patients and ISKDC grades in 17 (65\%), whereas chronicity score increased in 22 $(85 \%)$ patients and tubulointerstitial score in $21(81 \%)$. Changes occurred similarly in groups I and II (Fig. 1). In all five patients with unfavorable outcome, activity scores decreased whereas chronicity and tubulointerstitial scores increased (Table S2). SQC activity and chronicity scores in the follow-up biopsy showed no significant correlation with time from disease onset to treatment and did not differ significantly according to the initial treatment (data not shown). In multiple regression, activity score in the diagnostic biopsy was an independent predictor of the chronicity score in the follow-up biopsy (Table 3). Median number of glomeruli per biopsy was 21 (IQR 12-25) in the diagnostic biopsy and 15 (IQR 12-22) in the follow-up biopsy.

\section{MEST-C}

Table 4 shows MEST-C scores in the diagnostic and follow-up biopsies. Between the biopsies, $\mathrm{S} 1$ (from 38 to $79 \%$; $p=0.006$ ) and T1-2 (from 0 to $13 \% ; p=0.25$ ) scores increased, whereas $\mathrm{E} 1$ (from 83 to $13 \% ; p<0.001$ ) and $\mathrm{C} 1-2$ (from 63 to $25 \%$; $p=$ $0.022)$ scores decreased. M1 was similarly present in both biopsies $(33 \% ; p>0.99)$. Table 4 also reports biopsies where tubular atrophy and/or interstitial fibrosis ( $T$ score) was present, but for less than $25 \%$ of the cortical area (marked as $T>0 \%$ in 
Table 2 Patient characteristics at the follow-up renal biopsy, ISKDC findings of the follow-up biopsy, and outcome at the last follow-up visit

\begin{tabular}{|c|c|c|c|c|}
\hline & All $(n=26)$ & Group I $(n=11)$ & Group II $(n=15)$ & $p$ value \\
\hline \multicolumn{5}{|l|}{ At the follow-up biopsy } \\
\hline Age (years) & $12.6 \pm 4.6$ & $11.1 \pm 5.4$ & $13.8 \pm 3.7$ & 0.14 \\
\hline Plasma creatinine $(\mu \mathrm{mol} / \mathrm{L})$ & $58(41-67)$ & $53(38-58)$ & $66(53-74)$ & 0.024 \\
\hline eGFR $\left(\mathrm{mL} / \mathrm{min} / 1.73 \mathrm{~m}^{2}\right)$ & $98(89-121)$ & $103(96-126)$ & $94(82-108)$ & 0.069 \\
\hline dU-Prot ${ }^{\mathrm{a}}$ (g/day) & $0.3(0.1-1.2)$ & $0.10(0.91-0.11)$ & $0.9(0.3-3.3)$ & $\mathrm{NA}^{\mathrm{b}}$ \\
\hline Hematuria, $n(\%)$ & $16(62 \%)$ & $5(45 \%)$ & $11(73 \%)$ & 0.23 \\
\hline \multicolumn{5}{|l|}{ ISKDC } \\
\hline I & 1 & 1 & 0 & \\
\hline II & 16 & 9 & 7 & \\
\hline IIIa & 3 & 0 & 3 & \\
\hline IIIb & 4 & 1 & 3 & \\
\hline IV & 1 & 0 & 1 & \\
\hline $\mathrm{V}$ & 1 & 0 & 1 & \\
\hline SQC activity score & $2(1-2)$ & $1(1-2)$ & $2(1-4,5)$ & 0.033 \\
\hline SQC chronicity score & $7(5-9)$ & $6(3.5-7)$ & $9(7-10.5)$ & 0.013 \\
\hline SQC tubulointerstitial score & $3(2-3)$ & $3(1-3)$ & $3(2-4)$ & 0.096 \\
\hline \multicolumn{5}{|l|}{ At the last follow-up } \\
\hline Age (years) & $19.1 \pm 6.7$ & $16.0 \pm 6.5$ & $21.4 \pm 5.9$ & 0.035 \\
\hline Follow-up (years) & $8.6(6.4-11.3)$ & $6.8(6.2-8.2)$ & $9.0(8.3-12.8)$ & 0.041 \\
\hline Plasma creatinine $(\mu \mathrm{mol} / \mathrm{L})$ & $75(58-87)$ & $58(51-71)$ & $77(75-123)$ & 0.003 \\
\hline $\mathrm{eGFR}^{\mathrm{c}}\left(\mathrm{mL} / \mathrm{min} / 1.73 \mathrm{~m}^{2}\right)$ & $86(73-96)$ & $96(86-121)$ & $83(38-86)$ & 0.011 \\
\hline \multicolumn{5}{|l|}{ Outcome } \\
\hline A & 15 & 9 & 6 & \\
\hline $\mathrm{B}$ & 6 & 2 & 4 & \\
\hline $\mathrm{C}$ & 1 & 0 & 1 & \\
\hline $\mathrm{D}$ & 4 & 0 & 4 & \\
\hline
\end{tabular}

Group I represents patients without proteinuria and group II patients with proteinuria at the follow-up biopsy. eGFR, estimated glomerular filtration rate; $d U$-Prot, daily urine protein excretion; $N A$, not applicable

${ }^{\mathrm{a}} n=21$ ( 8 in group I and 13 in group II; for 3 patients in group I and 2 patients in group II proteinuria was measured only as a dipstick test or as U-Prot; these patients were not included in the analysis)

${ }^{\mathrm{b}}$ Group I comprises patients with no proteinuria at the follow-up renal biopsy

${ }^{\mathrm{c}} n=25$ (11 in group I and 14 in group II)

Table 3). $T>0 \%$ score increased from 4 to $67 \%$ between the diagnostic and follow-up biopsy $(p<0.001)$. Marked differences between group I and group II occurred especially in the follow-up biopsies' presence of C1-2 (0\% vs. 40\%, $p=0.052)$ and $\mathrm{E} 1(0 \%$ vs. $20 \%, p=0.27)$. Baseline characteristics for patients with and without $\mathrm{C} 1-2$ at the follow-up biopsy were: age 12.1 years (IQR 7.6-14.1) vs. 9.0 years (IQR 7.6-11.8) ( $p$ $=0.31)$, proteinuria $284 \mathrm{mg} / \mathrm{m}^{2} / \mathrm{h}($ IQR $106-336)$ vs. $131 \mathrm{mg} /$ $\mathrm{m}^{2} / \mathrm{h}(52-255)(p=0.35)$, and eGFR $94 \mathrm{~mL} / \mathrm{min} / 1.73 \mathrm{~m}^{2}$ (IQR $85-101)$ vs. $102 \mathrm{~mL} / \mathrm{min} / 1.73 \mathrm{~m}^{2}$ (IQR 89-110) $(p=0.42)$, respectively. Initial treatment of patients with $\mathrm{C} 1-2$ at the follow-up biopsy was methylprednisolone pulses $(n=4)$, cyclosporine A $(n=1)$, and cyclophosphamide $(n=1)$.

\section{Immunohistochemistry}

Expression of $\alpha$-SMA, vimentin, and PSGL- 1 was higher in HSN biopsies than in control specimens (combined HSN groups I and II vs. controls, $p<$ 0.001 for all markers). Tubulointerstitial scores $(\alpha-$ SMA, vimentin, PSGL-1) were higher in group I than in group II $(p<0.001$ for all markers) (Fig. 2). In glomeruli, PSGL-1 expression in groups I and II was similar $(p=0.85)$. Table 5 contains correlations between staining results and SQC activity, chronicity, and tubulointerstitial indices. $\alpha$-SMA showed positive correlations with all parameters and significant correlation with follow-up biopsies' tubulointerstitial index. Vimentin showed significant negative correlation with chronicity and tubulointerstitial index of the follow-up biopsy. PSGL-1 exhibited no significant correlation with the SQC indices. No staining showed significant correlation with the time from the onset of nephritis to the diagnostic renal biopsy ( $\alpha$-SMA: $r=0.22, p=0.38$; vimentin: $r=-0.066, p$ $=0.79$; PSGL-1 in glomeruli: $r=-0.13, p=0.62$; PSGL-1 in tubulointerstitium: $r=0.065, p=0.83$ ). 
Fig. 1 Changes in the activity (a), chronicity (b), and

tubulointerstitial scores (c) of the semiquantitative classification (SQC), and in the International Study of Kidney Diseases in Children classification (ISKDC) grades (d) between diagnostic and follow-up biopsy presented individually in 26 Henoch-Schönlein nephritis patients. The number of patients with decreased activity score $(p=0.62)$, decreased ISKDC grade $(p>0.99)$, increased chronicity score $(p>$ 0.99), or increased tubulointerstitial score $(p=0.62)$ did not differ between groups I and II. Numbers in figures denote the number of patients with similar change in respective SQC score or ISKDC grade a

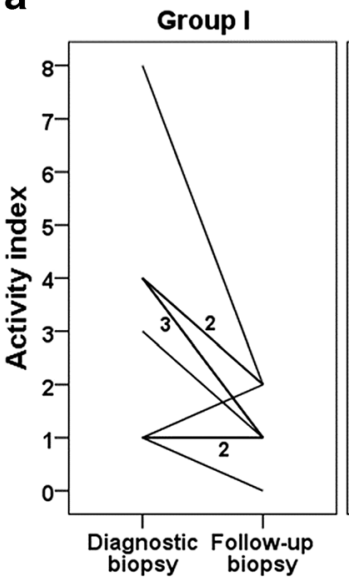

Group II

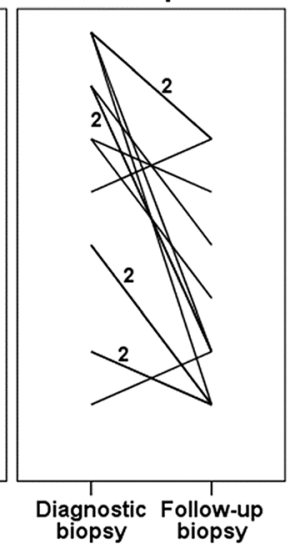

C

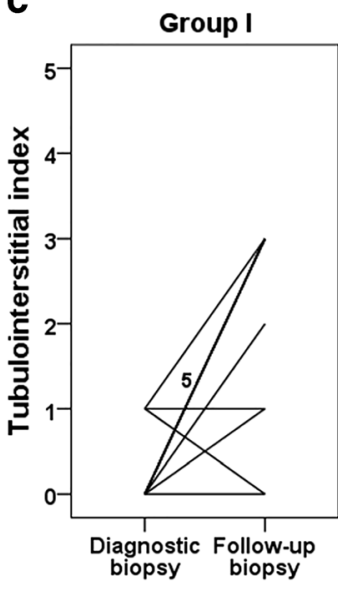

Group II

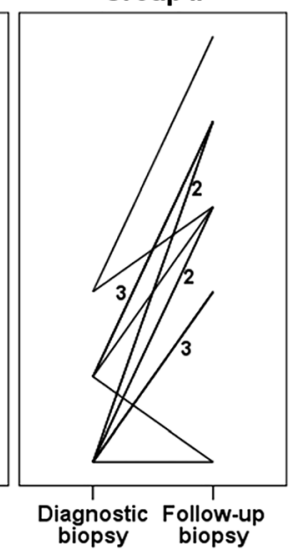

b

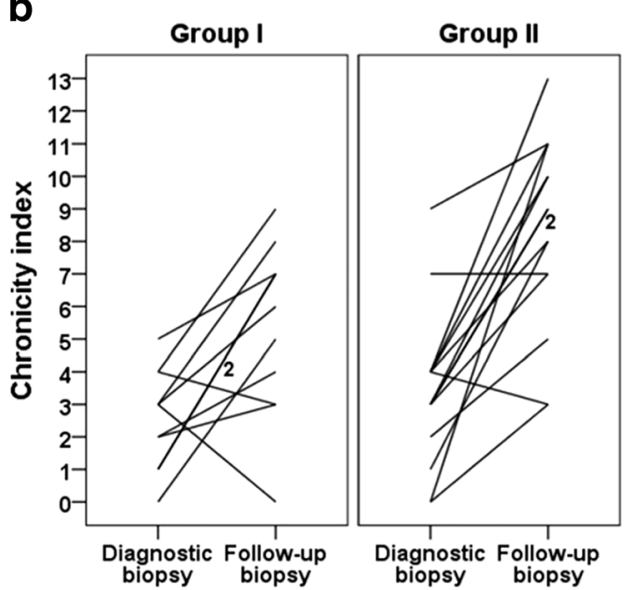

d

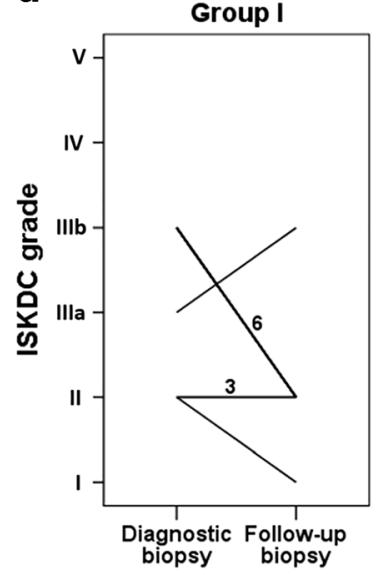

Group II

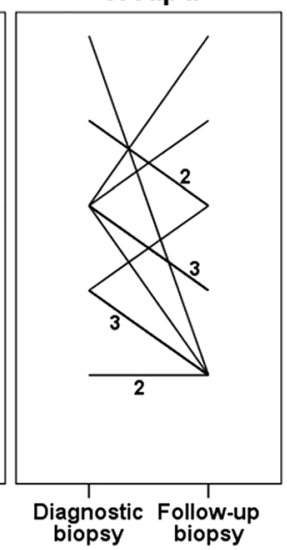

\section{Discussion}

The aim of our study was to evaluate the prognostic value of three histologic classifications: ISKDC, SQC, and MEST-C. We also evaluated whether pro-fibrotic and inflammatory markers in diagnostic renal biopsy specimens predicted clinical outcomes and lesions in follow-up renal biopsies. Between the diagnostic and follow-up biopsy, active changes decreased and chronic changes increased regardless of the clinical symptoms. Nonetheless, patients with proteinuria at the follow-up biopsy (group II) had higher SQC activity scores in the diagnostic biopsy and higher activity and chronicity scores at the follow-up biopsy than patients without proteinuria (group I). The studied pro-fibrotic and inflammatory markers were higher in HSN biopsy specimens than in controls, but showed no value as predictors of prolonged proteinuria.

We divided the patients according to proteinuria at the follow-up biopsy, since prolonged proteinuria is associated with worsened prognosis $[15,16]$. In accordance with these reports, all five patients with poor outcome in our study had prolonged proteinuria. In most patients, regardless of the proteinuria, activity scores decreased and chronicity scores increased from diagnostic biopsy to follow-up biopsy. This was also evident in MEST-C scores: between the biopsies E1 decreased, whereas $\mathrm{S} 1, \mathrm{~T} 1-2$, and $\mathrm{T}>0 \%$ increased. Regarding these changes in activity and chronicity scores in serial HSN biopsies, previous studies have shown comparable results [17-19]. Consistent with the findings of Shin et al. [17], the follow-up biopsies of 21 patients with favorable outcome contained chronic histologic changes. This suggests that even with favorable therapeutic outcome, HSN patients require long-term follow-up since the increased histological chronicity may impact the long-term prognosis. Furthermore, since most of our patients were treated with immunosuppressive drugs and angiotensin-converting enzyme inhibitors, progression of the chronicity signs can be considered as an insufficient response to the treatment.

Based on our results, MEST-C parameters in pediatric HSN seem feasible. MEST-C originates from adult IgAN patient data and thus some modifications to the classification may prove necessary for the evaluation of pediatric HSN. For example, tubulointerstitial lesions of MEST-C (i.e., T1 and 2) in our cohort were rare. They were, however, more common after inclusion of biopsies with less than $25 \%$ of affected area. Progression of tubulointerstitial lesions associates with a longer duration of proteinuria [20], and it could therefore prove 
Table 3 Linear regression results for chronicity score in the follow-up biopsy

\begin{tabular}{|c|c|c|c|c|c|c|}
\hline & \multicolumn{3}{|c|}{ Univariable } & \multicolumn{3}{|c|}{ Multivariable } \\
\hline & $B$ & $95 \% \mathrm{CI}$ & $p$ value & $B$ & $95 \% \mathrm{CI}$ & $p$ value \\
\hline Gender (male vs. female) & -0.39 & -3.12 to 2.34 & 0.77 & & & \\
\hline Age at onset (years) & 0.35 & -0.19 to 0.71 & 0.062 & -0.005 & -0.30 to 0.29 & 0.97 \\
\hline dU-Prot $^{\mathrm{a}}\left(\mathrm{mg} / \mathrm{h} / \mathrm{m}^{2}\right)$ & -0.004 & -0.014 to 0.005 & 0.33 & & & \\
\hline $\mathrm{eGFR}^{\mathrm{a}}\left(\mathrm{mL} / \mathrm{min} / 1.73 \mathrm{~m}^{2}\right)$ & -0.011 & -0.064 to 0.041 & 0.66 & & & \\
\hline SQC activity score ${ }^{\mathrm{a}}$ & 0.94 & 0.60 to 1.28 & $<0.001$ & 0.74 & 0.34 to 1.12 & 0.001 \\
\hline SQC chronicity score ${ }^{\mathrm{a}}$ & 0.43 & -0.19 to 1.05 & 0.17 & 0.42 & -0.032 to 0.88 & 0.067 \\
\hline $\mathrm{M}^{\mathrm{a}}$ & 0 & -2.68 to 2.68 & $>0.99$ & & & \\
\hline $\mathrm{E} 1^{\mathrm{a}}$ & -1.35 & -4.68 to 1.98 & 0.41 & & & \\
\hline $\mathrm{S} 1^{\mathrm{a}}$ & 0.11 & -2.50 to 2.72 & 0.93 & & & \\
\hline $\mathrm{C} 1-2^{\mathrm{a}}$ & 2.38 & -0.008 to 4.76 & 0.051 & 0.86 & -1.23 to 2.95 & 0.40 \\
\hline
\end{tabular}

T1 was not present in the diagnostic biopsies and thus was not included in the analyses. Since crescents are included also in the SQC activity score, we performed multivariable analysis with age at onset, SQC activity score, and SQC chronicity score (i.e., excluding C1-2 from the analysis). Results did not markedly change (data not shown). $B$, regression coefficient; $C I$, confidence interval; $d U$-Prot, daily urine protein excretion; eGFR, estimated glomerular filtration rate; $M$, mesangial hypercellularity; $E$, endocapillary proliferation; $S$, segmental glomeruloscrelosis; $T$, tubular atrophy and/or interstitial fibrosis; $C$, crescents

${ }^{\mathrm{a}}$ At the diagnostic biopsy

important to consider all lesions affecting the tubulointerstitium and not only those exceeding $25 \%$ of the renal cortex area.

C1-2 were present in both groups' diagnostic biopsies, but were only seen in the follow-up biopsies in group II. The persistence of crescents thus seems to associate with prolonged proteinuria, supporting results from other studies that treatment should be commenced before fibrotization of crescents [19]. Active treatment is also supported by the finding that active lesions in the diagnostic biopsy predicted chronic lesions in the follow-up biopsy. M1 was similarly present in both biopsies. Similar results were observed in adult IgAN patients after a 6-month treatment with mycophenolate mofetil and prednisone [21]. M1 thus seem not to be a flexible sign of clinical improvement and may suggest that the underlying pathogenetic event is still present. In HSN, the clinical meaning (and whether they need further therapy) of the persistent M1 and increased S1/T1-2 findings is unknown. It is possible that the renal damage continues despite minimal clinical signs and that the eGFR has remained stable due to the insufficient follow-up.

Oxford classification has been validated to predict outcomes in IgAN, and in a recent study, M1, S1, and T1-2 were predictive of progression decades after the renal biopsy [22]. Previous studies on the feasibility of MEST-C in pediatric

Table 4 MEST-C scoring according to the Oxford classification

\begin{tabular}{|c|c|c|c|c|c|c|c|c|}
\hline & \multicolumn{4}{|c|}{ Diagnostic biopsy } & \multicolumn{4}{|c|}{ Follow-up biopsy } \\
\hline & All & Group I & Group II & $p$ value & All & Group I & Group II & $p$ value \\
\hline $\mathrm{M} 1, n(\%)$ & $8(33 \%)$ & $2(22 \%)$ & $6(40 \%)$ & 0.66 & $8(33 \%)$ & $2(22 \%)$ & $6(40 \%)$ & 0.66 \\
\hline $\mathrm{E} 1, n(\%)$ & $20(83 \%)$ & $7(78 \%)$ & $13(87 \%)$ & 0.62 & $3(13 \%)$ & 0 & $3(20 \%)$ & 0.27 \\
\hline $\mathrm{S} 1, n(\%)$ & $9(38 \%)$ & $4(44 \%)$ & $5(33 \%)$ & 0.68 & $19(79 \%)$ & $6(67 \%)$ & $13(87 \%)$ & 0.33 \\
\hline $\mathrm{T} 1-2, n(\%)$ & 0 & 0 & 0 & NA & $3(13 \%)$ & $1(11 \%)$ & $2(13 \%)$ & $>0.99$ \\
\hline $\mathrm{T}>0 \%^{\mathrm{a}}, n(\%)$ & $1(4 \%)$ & 0 & $1(7 \%)$ & $>0.99$ & $16(67 \%)$ & $5(56 \%)$ & $11(73 \%)$ & 0.41 \\
\hline $\mathrm{C} 1, n(\%)$ & $9(38 \%)$ & $5(56 \%)$ & $4(27 \%)$ & 0.21 & $5(21 \%)$ & 0 & $5(33 \%)$ & 0.12 \\
\hline $\mathrm{C} 2, n(\%)$ & $6(25 \%)$ & 0 & $6(40 \%)$ & 0.052 & $1(4 \%)$ & 0 & $1(7 \%)$ & $>0.99$ \\
\hline $\mathrm{C} 1-2, n(\%)$ & $15(63 \%)$ & $5(56 \%)$ & $10(67 \%)$ & 0.68 & $6(25 \%)$ & 0 & $6(40 \%)$ & 0.052 \\
\hline Total MEST-C score & $2.5(2-3)$ & $2(2-3)$ & $3(2-3)$ & 0.39 & $1(1-2.5)$ & $1(1-1)$ & $2(1-3.5)$ & 0.10 \\
\hline
\end{tabular}

48/52 renal biopsy specimens (combined diagnostic and follow-up renal biopsy) were available for MEST-C analysis. Group I represents patients without proteinuria and group II patients with proteinuria at the follow-up biopsy. $M$, mesangial hypercellularity; $E$, endocapillary proliferation; $S$, segmental glomeruloscrelosis; $T$, tubular atrophy and/or interstitial fibrosis; $C$, crescents; $N A$, not applicable

${ }^{a}$ Comprises patients whose renal biopsies showed tubular atrophy and/or interstitial fibrosis affecting $>0 \%$ of the renal cortical area 


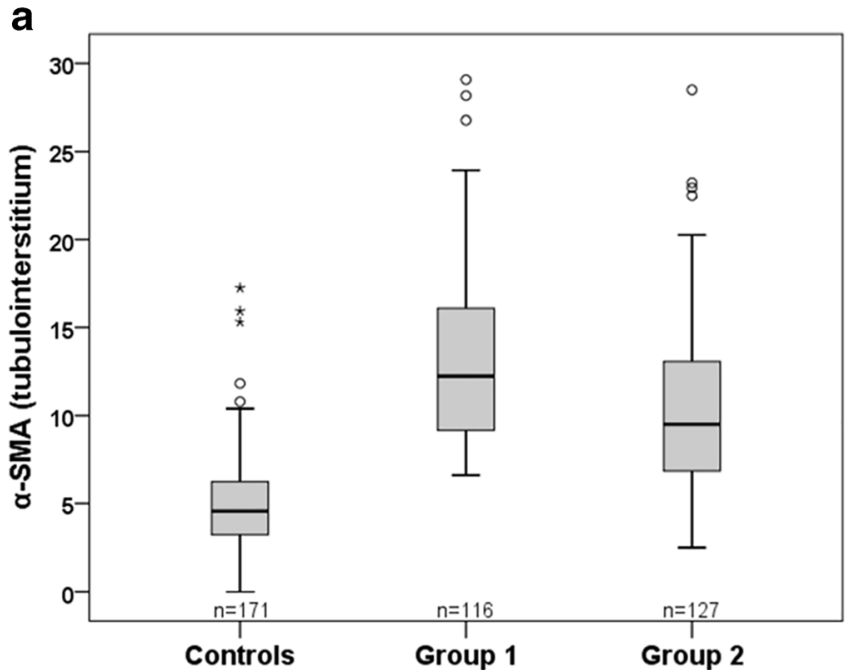

b

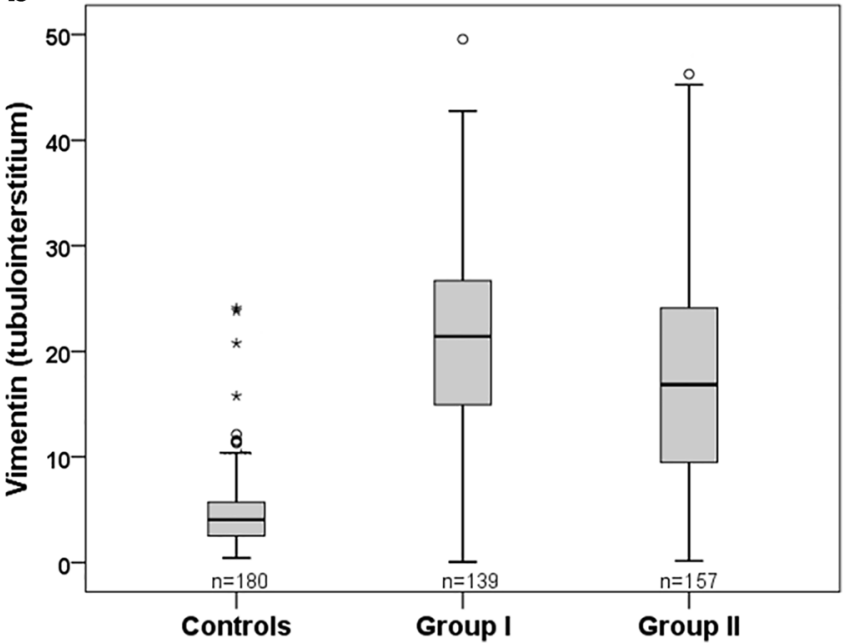

C

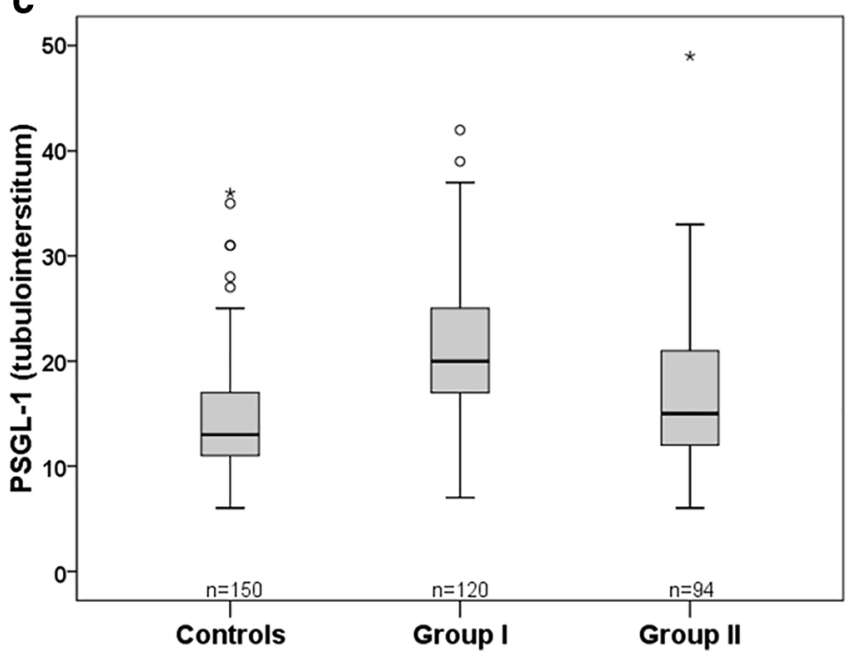

d

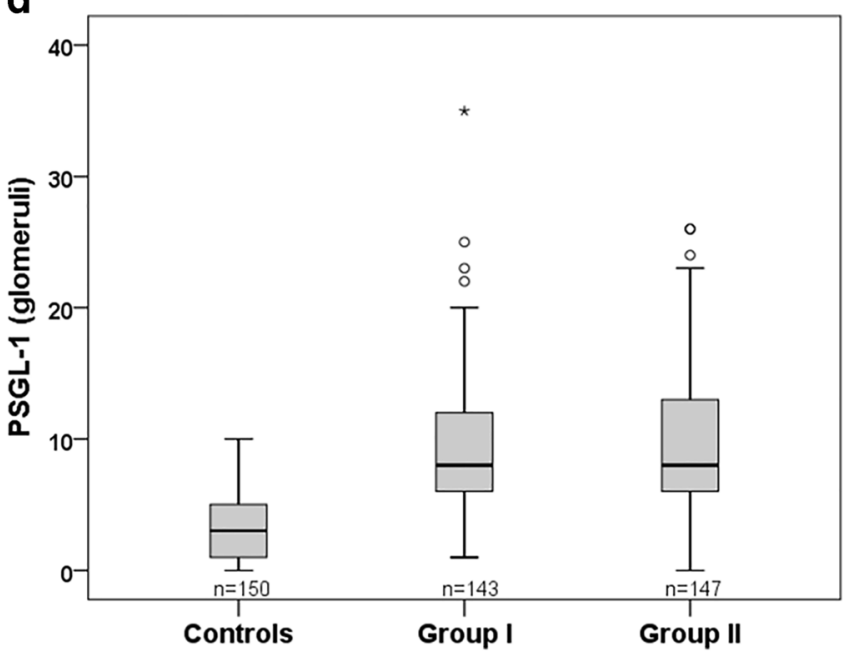

Fig. 2 Scores for tubulointerstitial alpha-smooth muscle actin ( $\alpha$-SMA) (a), vimentin (b), and P-selectin glycoprotein ligand-1 (PSGL-1) (c), as well as for glomerular PSGL-1 (d), in normal kidney specimens and in Henoch-Schönlein nephritis patients without proteinuria (group I) and

with proteinuria (group II) at the follow-up renal biopsy. Numbers in figures denote the total number of microscopic fields or glomeruli in each group

Table 5 Correlation with mean expression of $\alpha$-SMA, vimentin, and PSGL-1 scores in the diagnostic biopsy and activity, chronicity, and tubulointerstitial indices of the diagnostic and follow-up renal biopsy

\begin{tabular}{|c|c|c|c|c|c|c|}
\hline & \multicolumn{3}{|c|}{ Diagnostic biopsy } & \multicolumn{3}{|c|}{ Follow-up biopsy } \\
\hline & AI & $\mathrm{CI}$ & TI & $\mathrm{AI}$ & $\mathrm{CI}$ & $\mathrm{TI}$ \\
\hline$\alpha$-SMA in tubulointerstitium & $\begin{array}{l}r=0.26 \\
p=0.29\end{array}$ & $\begin{array}{l}r=0.27 \\
p=0.29\end{array}$ & $\begin{array}{l}r=0.37 \\
p=0.13\end{array}$ & $\begin{array}{l}r=0.35 \\
p=0.15\end{array}$ & $\begin{array}{l}r=0.27 \\
p=0.29\end{array}$ & $\begin{array}{l}r=0.55 \\
p=0.019\end{array}$ \\
\hline Vimentin in tubulointerstitium & $\begin{array}{l}r=-0.34 \\
p=0.15\end{array}$ & $\begin{array}{l}r=-0.37 \\
p=0.12\end{array}$ & $\begin{array}{l}r=-0.27 \\
p=0.27\end{array}$ & $\begin{array}{l}r=-0.27 \\
p=0.26\end{array}$ & $\begin{array}{l}r=-0.60 \\
p=0.007\end{array}$ & $\begin{array}{l}r=-0.53 \\
p=0.019\end{array}$ \\
\hline PSGL-1 in tubulointerstitium & $\begin{array}{l}r=0.081 \\
p=0.78\end{array}$ & $\begin{array}{l}r=0.067 \\
p=0.82\end{array}$ & $\begin{array}{l}r=0.13 \\
p=0.65\end{array}$ & $\begin{array}{l}r=-0.012 \\
p=0.97\end{array}$ & $\begin{array}{l}r=-0.091 \\
p=0.76\end{array}$ & $\begin{array}{l}r=-0.044 \\
p=0.88\end{array}$ \\
\hline PSGL-1 in glomeruli & $\begin{aligned} r & =-0.10 \\
p & =0.70\end{aligned}$ & $\begin{array}{l}r=0.26 \\
p=0.32\end{array}$ & $\begin{array}{l}r=0.06 \\
p=0.82\end{array}$ & $\begin{array}{l}r=-0.02 \\
p=0.94\end{array}$ & $\begin{array}{l}r=-0.16 \\
p=0.53\end{array}$ & $\begin{array}{l}r=-0.36 \\
p=0.15\end{array}$ \\
\hline
\end{tabular}

$A I$, activity index; $C I$, chronicity index; $T I$, tubulointerstitial index; $\alpha$-SMA, alpha-smooth muscle actin; PSGL-1, P-selectin glycoprotein ligand-1 
HSN have suggested that the chronic lesions (S1 and T1-2) correlate with poor outcome. Xu et al. reported that segmental glomerulosclerosis (S1) was associated with a poor renal outcome in univariate analysis, whereas no significant association existed with other parameters. T1 and 2 lesions were, however, negatively associated with the risk of proteinuria remission while other lesions showed no significant association. Interestingly, the number of biopsies with especially E1 and $\mathrm{T} 1$ and 2 in the study by Xu et al. [23] differs from ours. This may reflect inter-observer variation between pathologists or differences in patient material and biopsy timing. T1-2 and $\mathrm{S} 1$ associated with worsened renal survival also in a recent study of 75 biopsied pediatric HSN patients [24]. In two studies with adult HSN patients, E1 also predicted outcome, with or without $\mathrm{S} 1[25,26]$. In our study, marked differences between groups I and II occurred in C1-2 and E1 in the followup biopsy, although no MEST-C parameter differed significantly. Significant differences existed, however, in the SQC subclasses as activity indices in both biopsies and chronicity index in the follow-up biopsy were higher in proteinuric patients (group II) than in non-proteinuric patients (group I). This may indicate that the evaluation of several histologic variables together could be more advantageous than the assessment of single separate variables.

Vimentin and $\alpha$-SMA are markers of fibroblast activation [27], whereas PSGL-1 is an inflammatory cell marker [28]. Vimentin and $\alpha$-SMA associate with disease progression in IgAN [29], but reports on the expression of these molecules in HSN are scarce. Kawasaki et al. studied the expression of $\alpha$ SMA and c-MET in HSN. Hepatocyte growth factor, which has anti-fibrogenetic effects, uses c-MET as a receptor. In the study by Kawasaki et al., the expression of $\alpha$-SMA in the first renal biopsy correlated with the chronicity index of the second biopsy in patients with crescents (ISKDC $\geq$ III) in the first renal biopsy. c-MET showed no significant correlation with activity or chronicity indices [18]. Our results are consistent with those of Kawasaki et al. concerning $\alpha$-SMA since in our study, tubulointerstitial $\alpha$-SMA had significant positive correlation with the tubulointerstitial index - which reflects mainly chronic changes - of the follow-up biopsy. Nonetheless, $\alpha$ SMA showed no significant correlation with the chronicity index itself. Tubulointerstitial scores for $\alpha$-SMA, vimentin, and PSGL-1 were surprisingly higher in patients without proteinuria at the follow-up biopsy, but all three markers showed great overlap between the HSN groups (Fig. 2). We were not able to analyze the expression of $\alpha$-SMA and vimentin from glomeruli. This was due to heavy staining, which precluded any quantitative or semiquantitative analysis.

Our study has limitations. First, most patients had received therapy, which had most likely altered the disease course and possibly hampered the prognostic significance of renal biopsies overall [30]. Second, the low number of patients with unfavorable outcome limits the interpretation of results from comparison between patients with favorable and unfavorable outcome. These two limitations are probably due to the active treatment of severe HSN in Finland [31]. Third, histologic evaluation is always subject to inter-rater variability. The inter-rater variability of SQC, assessed in our previous study, showed fair to good reproducibility in a randomly chosen subset of ten biopsies [10].

Our study has strengths. First, we were able to analyze follow-up biopsies from patients with and without proteinuria at the follow-up biopsy. This allowed us to evaluate the biopsies in relation to the patients' clinical status. Second, data on the feasibility of the MEST-C in pediatric HSN is scarce, and in our study, the follow-up biopsies also underwent evaluation with MEST-C classification. Third, the median follow-up time in our study was 8.6 years.

Our results suggest that from diagnostic to follow-up renal biopsy, active signs decrease and chronic signs increase regardless of the clinical status, and thus follow-up biopsies provide limited additive information to the ongoing clinical symptoms (especially severe proteinuria) in HSN outcome prediction. Nonetheless, all severe HSN patients, even after a good therapeutic result, require long-term follow-up. Compared to control specimens, HSN biopsies showed overexpression of the pro-fibrotic and inflammatory markers, but none were associated with prolonged proteinuria.

Acknowledgments Authors thank Tuike Helmiö for her excellent technical assistance with the immunohistochemical stainings.

Funding information Open access funding provided by University of Helsinki including Helsinki University Central Hospital. The Finnish Kidney Foundation, the Foundation for Pediatric Research, the Orion Research Foundation, and the Alma and K.A. Snellman Foundation, Oulu, Finland supported this study by a grant to M.K.

Compliance with ethical standards This study was approved by the ethics committee of Helsinki University Hospital (164/13/03/03/ 2016).

Conflicts of interest The authors declare that they have no conflict of interest.

Open Access This article is distributed under the terms of the Creative Commons Attribution 4.0 International License (http:// creativecommons.org/licenses/by/4.0/), which permits unrestricted use, distribution, and reproduction in any medium, provided you give appropriate credit to the original author(s) and the source, provide a link to the Creative Commons license, and indicate if changes were made.

\section{References}

1. Gardner-Medwin JM, Dolezalova P, Cummins C, Southwood TR (2002) Incidence of Henoch-Schönlein purpura, Kawasaki disease, and rare vasculitides in children of different ethnic origins. Lancet 360:1197-1202 
2. Narchi H (2005) Risk of long term renal impairment and duration of follow up recommended for Henoch-Schönlein purpura with normal or minimal urinary findings: a systematic review. Arch Dis Child 90:916-920

3. Ronkainen J, Nuutinen M, Koskimies O (2002) The adult kidney 24 years after childhood Henoch-Schönlein purpura: a retrospective cohort study. Lancet 360:666-670

4. Goldstein AR, White RH, Akuse R, Chantler C (1992) Long-term follow-up of childhood Henoch-Schönlein nephritis. Lancet 339: 280-282

5. Counahan R, Winterborn MH, White RH, Heaton JM, Meadow SR, Bluett NH, Swetschin H, Cameron JS, Chantler C (1977) Prognosis of Henoch-Schönlein nephritis in children. Br Med J 2:11-14

6. Davin JC, Coppo R (2014) Henoch-Schönlein purpura nephritis in children. Nat Rev Nephrol 10:563-573

7. Jelusic M, Sestan M, Cimaz R, Ozen S (2019) Different histological classifications for Henoch-Schönlein purpura nephritis: which one should be used? Pediatr Rheumatol Online J 17:10-019-0311-z

8. Working Group of the International IgA Nephropathy Network and the Renal Pathology Society, Roberts IS, Cook HT, Troyanov S, Alpers CE, Amore A, Barratt J, Berthoux F, Bonsib S, Bruijn JA, Cattran DC, Coppo R, D'Agati V, D'Amico G, Emancipator S, Emma F, Feehally J, Ferrario F, Fervenza FC, Florquin S, Fogo A, Geddes CC, Groene HJ, Haas M, Herzenberg AM, Hill PA, Hogg RJ, Hsu SI, Jennette JC, Joh K, Julian BA, Kawamura T, Lai FM, Li LS, Li PK, Liu ZH, Mackinnon B, Mezzano S, Schena FP, Tomino Y, Walker PD, Wang H, Weening JJ, Yoshikawa N, Zhang H (2009) The Oxford classification of IgA nephropathy: pathology definitions, correlations, and reproducibility. Kidney Int 76:546-556

9. Trimarchi H, Barratt J, Cattran DC, Cook HT, Coppo R, Haas M, Liu ZH, Roberts IS, Yuzawa Y, Zhang H, Feehally J, IgAN Classification Working Group of the International IgA Nephropathy Network and the Renal Pathology Society, Conference Participants (2017) Oxford classification of IgA nephropathy 2016: an update from the IgA nephropathy classification working group. Kidney Int 91:1014-1021

10. Koskela M, Ylinen E, Ukonmaanaho EM, Autio-Harmainen H, Heikkila P, Lohi J, Jauhola O, Ronkainen J, Jahnukainen T, Nuutinen M (2017) The ISKDC classification and a new semiquantitative classification for predicting outcomes of Henoch-Schönlein purpura nephritis. Pediatr Nephrol 32:1201-1209

11. Jauhola O, Ronkainen J, Autio-Harmainen H, Koskimies O, AlaHouhala M, Arikoski P, Holtta T, Jahnukainen T, Rajantie J, Ormala T, Nuutinen M (2011) Cyclosporine A vs. methylprednisolone for Henoch-Schönlein nephritis: a randomized trial. Pediatr Nephrol 26:2159-2166

12. Kidney disease: Improving global outcomes (KDIGO) CKD work group. KDIGO 2012 clinical practice guideline for the evaluation and management of chronic kidney disease. (2013) Kidney Int Suppl 3:1-150

13. Schwartz GJ, Munoz A, Schneider MF, Mak RH, Kaskel F, Warady BA, Furth SL (2009) New equations to estimate GFR in children with CKD. J Am Soc Nephrol 20:629-637

14. Nuutinen M, Lautala P, Remes M, Uhari M (2000) Nephrectomy in severe hypertension. Clin Nephrol 54:342-346

15. Coppo R, Andrulli S, Amore A, Gianoglio B, Conti G, Peruzzi L, Locatelli F, Cagnoli L (2006) Predictors of outcome in HenochSchönlein nephritis in children and adults. Am J Kidney Dis 47: 993-1003

16. Edstrom Halling S, Soderberg MP, Berg UB (2010) Predictors of outcome in Henoch-Schönlein nephritis. Pediatr Nephrol 25:1101-1108

17. Shin J, Park JM, Kim JH, Lee JS, Jeong HJ (2006) Factors affecting histological regression of crescentic Henoch-Schönlein nephritis in children. Pediatr Nephrol 21:54-59

18. Kawasaki Y, Imaizumi T, Matsuura H, Ohara S, Takano K, Suyama K, Hashimoto K, Nozawa R, Suzuki H, Hosoya M (2008) Renal expression of alpha-smooth muscle actin and c-met in children with Henoch-Schönlein purpura nephritis. Pediatr Nephrol 23:913-919

19. Niaudet P, Habib R (1998) Methylprednisolone pulse therapy in the treatment of severe forms of Schönlein-Henoch purpura nephritis. Pediatr Nephrol 12:238-243

20. Lim BJ, Shin JI, Choi SE, Rhim H, Lee JS, Kim PK, Jeong HJ, Kim JH (2016) The significance of tubulointerstitial lesions in childhood Henoch-Schönlein nephritis. Pediatr Nephrol 31:2087-2093

21. Hou JH, Le WB, Chen N, Wang WM, Liu ZS, Liu D, Chen JH, Tian J, Fu P, Hu ZX, Zeng CH, Liang SS, Zhou ML, Zhang HT, Liu ZH (2017) Mycophenolate mofetil combined with prednisone versus fulldose prednisone in IgA nephropathy with active proliferative lesions: a randomized controlled trial. Am J Kidney Dis 69:788-795

22. Coppo R, D'Arrigo G, Tripepi G, Russo ML, Roberts ISD, Bellur S, Cattran D, Cook TH, Feehally J, Tesar V, Maixnerova D, Peruzzi L, Amore A, Lundberg S, Di Palma AM, Gesualdo L, Emma F, Rollino C, Praga M, Biancone L, Pani A, Feriozzi S, Polci R, Barratt J, Del Vecchio L, Locatelli F, Pierucci A, Caliskan Y, Perkowska-Ptasinska A, Durlik M, Moggia E, Ballarin JC, Wetzels JFM, Goumenos D, Papasotiriou M, Galesic K, Toric L, Papagianni A, Stangou M, Benozzi L, Cusinato S, Berg U, Topaloglu R, Maggio M, OtsRosenberg M, D'Amico M, Geddes C, Balafa O, Quaglia M, Cravero R, Lino Cirami C, Fellstrom B, Floege J, Egido J, Mallamaci F, Zoccali C (2018) Is there long-term value of pathology scoring in immunoglobulin A nephropathy? A validation study of the oxford classification for IgA nephropathy (VALIGA) update. Nephrol Dial Transplant. https://doi.org/10.1093/ndt/gfy302

23. Xu K, Zhang L, Ding J, Wang S, Su B, Xiao H, Wang F, Zhong X, Li Y (2018) Value of the oxford classification of IgA nephropathy in children with Henoch-Schönlein purpura nephritis. J Nephrol 31: 279-286

24. Cakici EK, Gur G, Yazilitas F, Eroglu FK, Gungor T, Arda N, Orhan D, Ozalp Ates FS, Bulbul M (2019) A retrospective analysis of children with Henoch-Schönlein purpura and re-evaluation of renal pathologies using Oxford classification. Clin Exp Nephrol 23:939-947

25. Kim CH, Lim BJ, Bae YS, Kwon YE, Kim YL, Nam KH, Park KS, An SY, Koo HM, Doh FM, Lee MJ, Oh HJ, Yoo TH, Kang SW, Choi KH, Jeong HJ, Han SH (2014) Using the Oxford classification of IgA nephropathy to predict long-term outcomes of HenochSchönlein purpura nephritis in adults. Mod Pathol 27:972-982

26. Inagaki K, Kaihan AB, Hachiya A, Ozeki T, Ando M, Kato S, Yasuda Y, Maruyama S (2018) Clinical impact of endocapillary proliferation according to the Oxford classification among adults with Henoch-Schönlein purpura nephritis: a multicenter retrospective cohort study. BMC Nephrol 19:208-018-1009-z

27. Liu Y (2011) Cellular and molecular mechanisms of renal fibrosis. Nat Rev Nephrol 7:684-696

28. Ley K (2003) The role of selectins in inflammation and disease. Trends Mol Med 9:263-268

29. Yao J, Ke Z, Wang X, Peng F, Li B, Wu R (2014) Epithelialmesenchymal transition and apoptosis of renal tubular epithelial cells are associated with disease progression in patients with IgA nephropathy. Mol Med Rep 10:39-44

30. Ronkainen J, Ala-Houhala M, Huttunen NP, Jahnukainen T, Koskimies O, Ormala T, Nuutinen M (2003) Outcome of Henoch-Schöenlein nephritis with nephrotic-range proteinuria. Clin Nephrol 60:80-84

31. Koskela M, Jahnukainen T, Enden K, Arikoski P, Kataja J, Nuutinen M, Ylinen E (2019) Methylprednisolone or cyclosporine a in the treatment of Henoch-Schönlein nephritis: A nationwide study. Pediatr Nephrol 34:1447-1456

Publisher's note Springer Nature remains neutral with regard to jurisdictional claims in published maps and institutional affiliations. 